\title{
Development of Unilateral Piriformis Syndrome in a Female with Congenital Leg Length Discrepancy
}

\author{
Chia-Hung Sun, Shao-Chi Lu, Yung-Tsan Wu, Shin-Tsu Chang* \\ Department of Physical Medicine and Rehabilitation, Tri-Service General Hospital, School of Medicine, National Defense Medical \\ Center, Taipei, Chinese Taipei. \\ Email: *jim4882001@gmail.com
}

Received October $15^{\text {th }}$, 2012; revised November $15^{\text {th }}, 2012$; accepted November $22^{\text {nd }}, 2012$

\begin{abstract}
Background: This is a rare case of piriformis syndrome. We discuss the patient's symptoms, imaging study results, laboratory examination findings, and prescribed treatment in an attempt to determine the possible mechanisms or reasons for this patient's development of piriformis syndrome. Methods: The patient is a 22-year-old female soldier who was admitted for lower back pain with soreness radiating to her right buttock and right lower extremity. We found that she had leg length discrepancy under X-ray finding. She was diagnosed piriformis syndrome by physical examination and Magenetic Resonance Image. Results: We performed scanography for lower extremities revealed a left femur length of $42.7 \mathrm{~cm}$, a right femur length of $43.3 \mathrm{~cm}$, a left tibia length of $33.2 \mathrm{~cm}$, and a right tibia length of $33.6 \mathrm{~cm}$; her left lower extremity was $1 \mathrm{~cm}$ shorter than her right. The MRI of sacrum also reveals hypertrophy of right piriformis muscle. Conclusions: Piriformis syndrome might be caused by short-term compensation in congenital leg length discrepancy. The patient with leg length discrepancy changed posture when standing, walking, and running. These compensation postures could lead to abnormality position of bones where there is insertion of the muscle.
\end{abstract}

Keywords: Piriformis Syndrome; Leg Length Discrepancy; Visual Analogue Scale; Back Pain; Postural Compensation

\section{Introduction}

We report a case of congenital leg length discrepancy (LLD) that caused contralateral piriformis syndrome (PS). This is a rare case of PS. We discuss the patient's symptoms, imaging study results, laboratory examination findings, and prescribed treatment in an attempt to determine the possible mechanisms or reasons for this patient's development of PS. The patient had multiple characteristics commonly seen in PS, but her most prevalent complaint was lower back pain. We noted postural compensation in this patient, who had LLD which appeared to cause piriformis muscle spasm and contracture. After repeated irritation, she developed PS.

\section{Case Presentation}

The patient is a 22-year-old female soldier who was admitted for lower back pain with soreness radiating to her right buttock and right lower extremity. She did not have any systemic diseases or a history of any surgery or trauma, with the notable exception of having a congenital left lower limb congenitally shorter than the right lower limb. Her job intermittently involved working as a security

${ }^{*}$ Corresponding author. guard in her unit, which required her to stand for two 2 hours twice a day. The patient typically stands stood on her left leg and very rarely changes changed to her right leg, which she did only after maintaining a prolonged standing position. Despite her leg length discrepancy (LLD), she denied a history of leg pain. Approximately one week prior to presenting at the hospital, she experienced an acute pain in her right leg. This spasmodic pain was continuous and worsened over the week prior to presentation. She reported that the pain was an 8 - 9 on a Visual Analogue Score (VAS). On physical examination, a tender wedge-shaped mass was palpated in the anatomical region of the piriformis muscle. Other physical examination findings included a positive straight leg raise at $40^{\circ}$ degrees, and positive Patrick, Pace Abduction, Freiberg, Bonnet, and Saudek tests. There were no No abnormal findings were discovered on her L-spine Magenetic Resonance Image (MRI). Laboratory data were within normal limits. The scanography for lower extremities revealed a left femur length of $42.7 \mathrm{~cm}$, a right femur length of $43.3 \mathrm{~cm}$, a left tibia length of $33.2 \mathrm{~cm}$, and a right tibia length of $33.6 \mathrm{~cm}$ (Figure 1); her left lower extremity was $1 \mathrm{~cm}$ shorter than her right. The MRI of sacrum also reveals hypertrophy of piriformis muscle (Figure 2). Electromyography detected radiculo- 


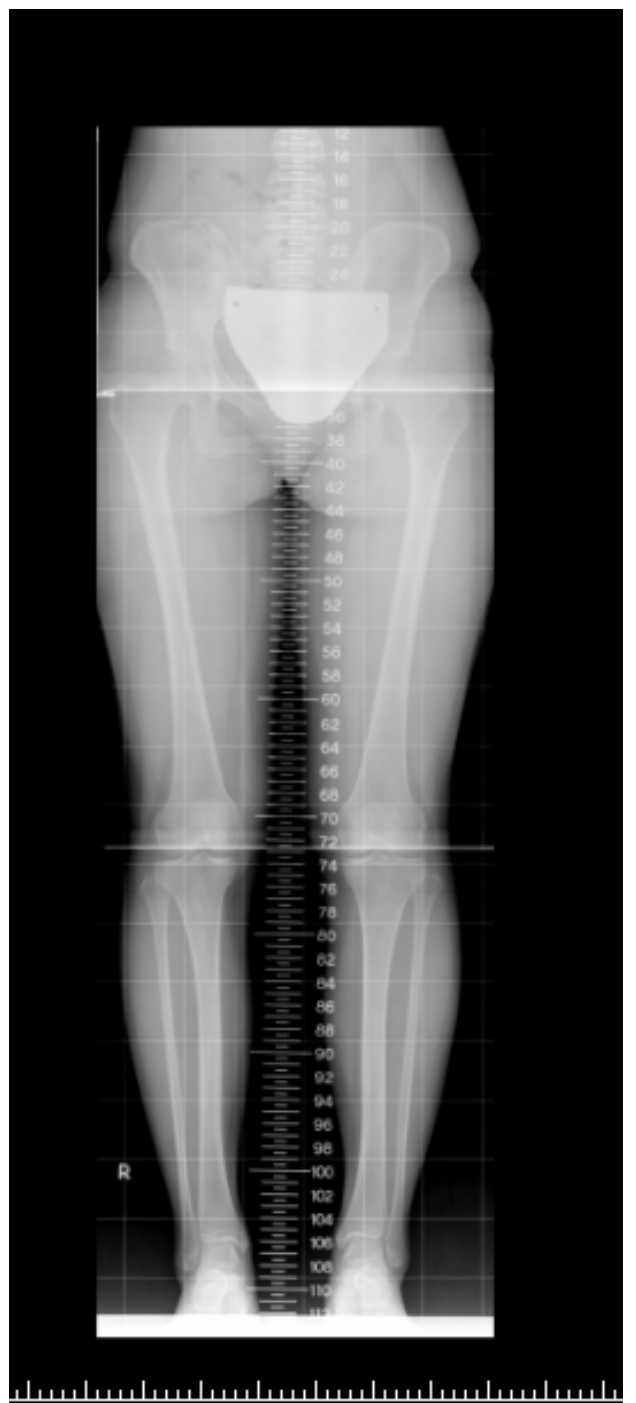

Figure 1. Scanography of femur length: Right $(\mathrm{Rt})=43.3$ $\mathrm{cm}$; Left $(\mathrm{Lt})=42.7 \mathrm{~cm}$; tibia length: $\mathrm{Rt}=33.6 \mathrm{~cm} ; \mathrm{Lt}=$ $33.2 \mathrm{~cm}$; left lower extremity shorter than the right.

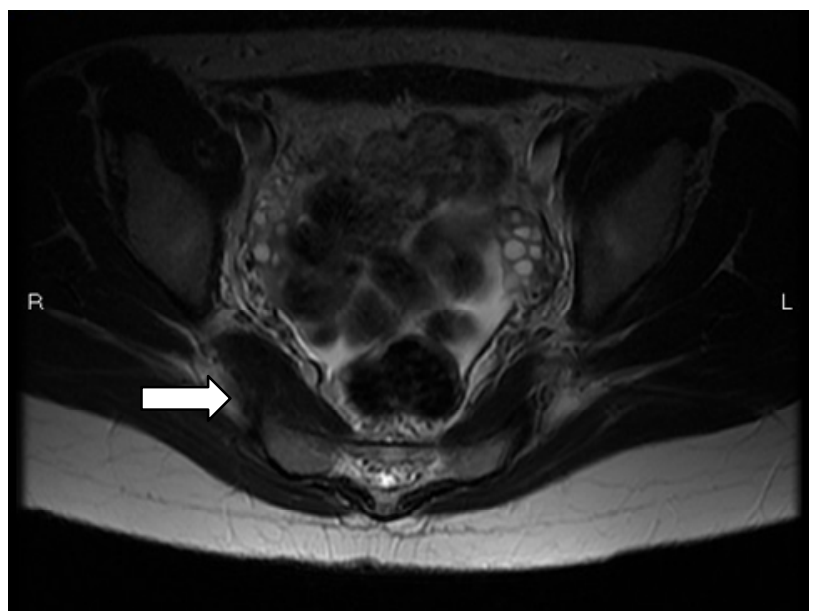

Figure 2. Hypertrophy of right piriformis muscle as arrow indication from Magenetic Resonance Image. pathy and some neurologic regeneration at the paravertebral spinal muscles innervated by L4, L5, and S1. A diagnosis of right-sided piriformis syndrome was made. Pain relievers, muscle relaxants (tramadol hydrochloride, acetaminophen, and baclofen), and rehabilitation improved the symptoms, resulting in an improvement of the VAS to scores of $2 \sim 3$ scores.

\section{Discussion}

Piriformis muscle syndrome [PS] was first described by Robinson in 1947. However some studies show that the proportion of PS is about $0.1 \%$ in orthopedic examination [1]. This is more common in women with a ratio of 3 to 1. PS presents an array of symptoms, from pain in the lower back, buttock, and perineal and inguinal areas to sciatica, and has been demonstrated to result from localized muscle spasm/contracture, local trauma at the buttocks, repetitive mechanical stressors, tilting sacral base/unstable pelvis, and local pressure overload in those with a thin shape [1]. Only one previous paper mentions that unilateral PS can be caused by LLD [2]. However, this paper noted that the PS developed homolateral to the shorter side, and not the taller side as in our case.

The piriformis muscle originates from the anterior surface of the lateral sacrum and fuses into a tendon inserting in the upper medial surface of the femoral greater trochanter. It is pyramidal or pear shaped and lies almost parallel to the posterior region of the gluteus medius muscle and extends through the greater sciatic foramen. It is innervated by the ventral rami of S1 and S2, which join to form the nerve to the piriformis. The sciatica nerve is composed of the ventral rami of L4 through S3 and it emerges through the greater sciatic foramen inferior to the piriformis muscle. With the leg extended, the piriformis muscle is mainly a hip external rotator, and when flexed, a hip abductor.

LLD can be categorized as congenital or acquired. Our patient had not experienced any major trauma, but had functional limitations when standing due to the imbalance and when walking for long periods. Studies on the effects of LLD on walking have found that gait asymmetries manifest throughout the kinetic chain. In general, an individual with an LLD must step down onto the short limb and vault over the long limb, resulting in an increase in vertical displacement of the centre of mass and hence an increase in energy consumption. In addition, an individual may shorten the longer leg by increasing pelvic obliquity, circumduction, increasing hip and/or knee flexion (steppage gait), increasing ankle dorsiflexion, or any combination of these. The person adopts these compensatory mechanisms to minimize the displacement of the body centre of mass during walking, thereby reducing energy expenditure. In this case, the piriformis muscle was in spasm and contracture during increasing pelvic 
obliquity, increasing hip circumduction to compensate.

LLD has been implicated in a variety of disorders, including lower back pain (LBP), scoliosis, pelvic and sacral malalignments, arthritis of the spine, hip pain and osteoarthritis, lower extremity stress fractures, aseptic loosening of the prosthetic hip, trochanteric bursitis, myofascial pain syndrome (MPS) of the peroneus longus, patellar apicitis, and meralgia paresthetica. Here, we report a rare case of piriformis syndrome caused by LLD and discuss the mechanisms for such an occurrence.

Short-term compensation for LLD in poor standing posture or balance can occur in many different ways. Some authors suggest that the longer leg often compensates with pronation of the foot on the longer leg, and the knee and hip can also compensate by flexion of the longer leg. If there is no compensation, the anterior and posterior iliac spines are lower on the side of the short leg, which in turn may result in a sacral base unleveling and/or scoliosis. In addition, a greater amount of pressure is transmitted through the hip of the longer leg due to both a decrease in the area of contact of the femoral head on the acetabulum and an increase in tone of the hip abductors secondary to an increased distance between origin and insertion [3]. The above reasons explain why such compensation may have caused piriformis muscle spasm and contracture in our case. We hypothesized that the contralateral piriformis syndrome was due to compensation in the longer side. The case was one of a congenital LLD causing contralateral piriformis syndrome. We investigated the mechanisms thereof, which have not been previously reported.

PS is diagnosed by physical examination such as the Pace Abduction, Freiberg, Bonnet, and Saudek tests. MRI neurography, CT, ultrasound, and EMG are also diagnostic tools. The pathology of piriformis muscle asymmetry or hypertrophy and sciatic nerve lesions at the sciatica notch can be found on MRI neurography [4]. CT and sonography can exclude other conditions but have less diagnostic sensitivity than MRI neurography. Nerve conduction velocity is normal in most cases but amplitude loss may be observed in the sensory nerve action potential or compound motor action potential, and EMG sometimes demonstrates delayed $\mathrm{H}$ reflex in the FAIR position [5].
Our EMG results were compatible with the above.

Hopayian and colleagues provided a review article discussing PS, and suggested that buttock pain is common, and more so than LBP [1]; PS-specific signs are the Freiberg sign and Pace sign, and limited SLR is the most common in routine testing for sciatica. Our case was compatible with most of the aforementioned symptoms, physical examinations, and EMG findings.

\section{Conclusion}

Piriformis syndrome might be caused by short-term compensation in congenital LLD, such as in the case reported here. The patient with LLD changed posture when standing, walking, and running. These compensation postures could lead to abnormality position of bones where there is insertion of the muscle. The muscle was in spasm and contracture due to these compensation pos- tures. From a theoretical viewpoint, it makes sense that congenital or acquired LLD may lead to the development of PS. Sciatica was apparent in both the herniated intervertebral disk and entrapment of the piriformis muscle. Postural rehabilitation should be performed to prevent recurrence of PS.

\section{REFERENCES}

[1] K. Hopayian, F. Song, R. Riera and S. Sambandan, "The Clinical Features of the Piriformis Syndrome: A Systemic Review," European Spine Journal, Vol. 19, No. 12, 2010, pp. 2095-2109. doi:10.1007/s00586-010-1504-9

[2] K. Dere, M. Akbas and N. Luleci, "A Rare Case of a Piriformis Syndrome,” Journal of Back Musculoskelet Rehabilitation, Vol. 22, No. 1, 2009, pp. 55-58.

[3] B. Gurney, "Leg Length Discrepancy,” Gait and Posture, Vol. 15, No. 2, 2002, pp. 195-206. doi:10.1016/S0966-6362(01)00148-5

[4] R. J. Halpin and A. Ganju, "Piriformis Syndrome: A Real Pain in the Buttock?” Neurosurgery, Vol. 65, No. 4, 2009, PP. A197-A202.

[5] R. M. Jawish, H. A. Assoum and C. F. Khamis, “Anatomical, Clinical, and Electrical Observations in Piriformis Syndrome," Journal of Orthopaedic Surgery and Research, Vol. 5, No. 3, 2010, pp. 1-7. 